\title{
A fauna apícola do Parque Municipal da Cachoeirinha (Iporá, GO)
}

\author{
Leandro Rodrigues Santiago ${ }^{1,3}$, Rute Magalhães Brito ${ }^{1}$, Thiago Mahlmann Vitoriano Lopes Muniz ${ }^{2}$, \\ Favízia Freitas de Oliveira ${ }^{2}$ \& Flávio de Oliveira Francisco ${ }^{1}$ \\ ${ }^{1}$ Unidade Universitária de Iporá, Universidade Estadual de Goiás - UEG, \\ Av. R2, Quadra 01, s/n, Jardim Novo Horizonte II, CEP 76200-000, Iporá, GO, Brasil, http://www.ueg.br \\ ${ }^{2}$ Departamento de Ciências Biológicas, Universidade Estadual de Feira de Santana - UEFS, \\ Av. Universitária, s/n, Km 03, BR 116 (Rod. Feira de Santana - Serrinha) \\ Cidade Universitária, CEP 44031-460, Feira de Santana, BA, Brasil, http://www.uefs.br \\ ${ }^{3}$ Autor para correspondência: Leandro Rodrigues Santiago, e-mail: leandrorsantiago@yahoo.com.br
}

SANTIAGO, L.R., BRITO, R.M., MUNIZ, T.M.V.L., OLIVEIRA, F.F. \& FRANCISCO, F.O. The bee fauna from Parque Municipal da Cachoeirinha (Iporá, Goiás state, Brazil). Biota Neotrop. 9(3): http://www.biotaneotropica.org.br/v9n3/en/abstract?short-communication+bn01509032009.

\begin{abstract}
Bees are dependent on flower nectar and pollen (main sources of protein and energy, respectively) and have an important economic and ecological role as pollinators, representing about 40 to $90 \%$ of phanerogams pollinators in several ecosystems. This work presents a description of bee species from Parque Municipal da Cachoeirinha in Iporá, Goiás state, Brazil. A total of 58 hours of capturing were performed on November and December 2004 and on March and May 2005. A total of 933 individuals were collected, distributed in one family, 16 tribes, 33 genera, and 62 species. Meliponini tribe presented the highest number of individuals and species. Most abundant species were Trigona chanchamayoensis (16.51\%), Tetragona clavipes $(14.79 \%)$ and Apis mellifera and Trigona recursa (12.33\%). This work presents the first occurrence in Goiás state for six genera and 12 species. Keywords: Hymenoptera, Cerrado, Apidae.
\end{abstract}

SANTIAGO, L.R., BRITO, R.M., MUNIZ, T.M.V.L., OLIVEIRA, F.F. \& FRANCISCO, F.O. A fauna apícola do Parque Municipal da Cachoeirinha (Iporá, GO). Biota Neotrop. 9(3): http://www.biotaneotropica.org.br/ v9n3/pt/abstract?short-communication+bn01509032009.

Resumo: As abelhas possuem grande importância econômica e ecológica, em vista de sua dependência dos recursos florais como o pólen e o néctar (principais fontes de proteínas e energia, respectivamente), representando cerca de 40 a $90 \%$ dos agentes polinizadores de fanerógamas em diversos ecossistemas. No presente trabalho, foi realizado o inventário apícola do Parque Municipal da Cachoeirinha (Iporá, GO). Foram realizadas 58 horas de coleta no total, realizadas nos meses de novembro/dezembro de 2004 e março e maio de 2005 . Foi coletado um total de 933 indivíduos, distribuídos em uma família, 16 tribos, 33 gêneros e 62 espécies. A tribo Meliponini foi a mais abundante em número de indivíduos e espécies. As espécies mais abundantes foram Trigona chanchamayoensis (16,51\%), Tetragona clavipes (14,79\%) e Apis mellifera e Trigona recursa (12,33\%). Este trabalho apresenta a primeira ocorrência no estado de Goiás para seis gêneros e 12 espécies.

Palavras-chave: Hymenoptera, Cerrado, Apidae. 


\section{Introdução}

As abelhas estão distribuídas em regiões temperadas e tropicais por todo o planeta. Apesar de existirem em torno de 16 mil espécies descritas, estima-se que o número real possa chegar a cerca de $30 \mathrm{mil}$ espécies (Michener 2000). A principal importância ecológica desses insetos é representada pelo seu papel na polinização das angiospermas, devido à sua dependência do néctar e o do pólen das flores como suas principais fontes de proteínas e energia, respectivamente (Nogueira-Neto 1997, Michener 2000). As abelhas estão diretamente ligadas ao ecossistema onde ocorrem, e são responsáveis por cerca de 40 a $90 \%$ da polinização de fanerógamas (Kerr et al. 1996), atuando, portanto, na manutenção do fluxo gênico e diversidade genética vegetal.

O bioma Cerrado é o segundo mais representativo do país e, apesar de bastante fragmentado, as abelhas se apresentam como os seus principais polinizadores (Anacleto \& Marchini 2005, d'Avila \& Marchini 2008, Ratter et al. 1997). Comparando-se a apifauna deste com outros biomas, nota-se que as abelhas do Cerrado apresentam grande riqueza em número de espécies e indivíduos, apresentando ainda variação na composição das espécies relacionada à variação florística, além de serem observadas muitas espécies raras (Silveira \& Campos 1995). Uma análise faunística realizada em um fragmento de Cerradão no estado de São Paulo mostra que, de todos os Hymenoptera visitantes florais coletados, 91,7\% eram abelhas (d'Avila e Marchini 2008).

Apesar de uma parte significativa do Cerrado brasileiro estar localizada no estado de Goiás, na literatura existem pouquíssimos estudos de abelhas realizados nessa região, sendo os mais próximos àqueles realizados em Brasília-DF (Boaventura 1998), Uberlândia-MG (Carvalho \& Bego 1996), Cassilândia-MS (Vieira et al. 2008) entre outros realizados no estado de São Paulo (Pedro 1992, Rêgo \& Brito 1996, Mateus 1998, Anacleto \& Marchini 2005, Andena et al. 2005, Silva et al. 2005).

O inventário da apifauna constitui-se no primeiro passo para o desenvolvimento de projetos específicos, como elaboração de estratégias de conservação, exploração racional, entre outros (Santos et al. 2004), além de serem importantes para a expansão do conhecimento já existente, pois produzem dados sobre a diversidade e distribuição geográfica de abelhas, necessários para análises de diversidade apícola como um todo (Silveira \& Godínez 1996). Em virtude disso, o presente trabalho teve por objetivo ampliar os conhecimentos a respeito da apifauna de uma região de Cerrado do estado de Goiás, através do inventariamento das espécies de abelhas do Parque Municipal da Cachoeirinha situado no município de Iporá.

\section{Material e Métodos}

\section{1. Área de estudo}

O trabalho foi realizado no Parque Municipal da Cachoeirinha

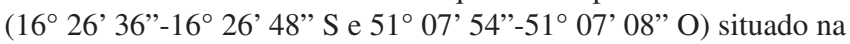
vila José Cândido Vieira no município de Iporá, Estado de Goiás, Brasil (Cunha et al. 2007). O município de Iporá (Figura 1) apresenta aproximadamente 400 a $800 \mathrm{~m}$ de altitude com clima tropical predominante e vegetação típica de Cerrado (Longhi et al. 2005). A temperatura média é de $29^{\circ} \mathrm{C}$ e as médias de precipitação são de aproximadamente 1.200 a $1.600 \mathrm{~mm}$, com estações seca (outono e inverno) e chuvosa (primavera e verão) bem definidas (Longhi et al. 2005). A área total do parque é de aproximadamente 5,8 ha, apresentando remanescentes de mata ciliar nas margens dos córregos Tamanduá e ribeirão Santo Antônio e mata seca semidecídua, embora não haja mais detalhes sobre sua constituição fitofisionômica (Cristofoli \& Leão 2005, Cunha et al. 2007). A área sofre muita ação

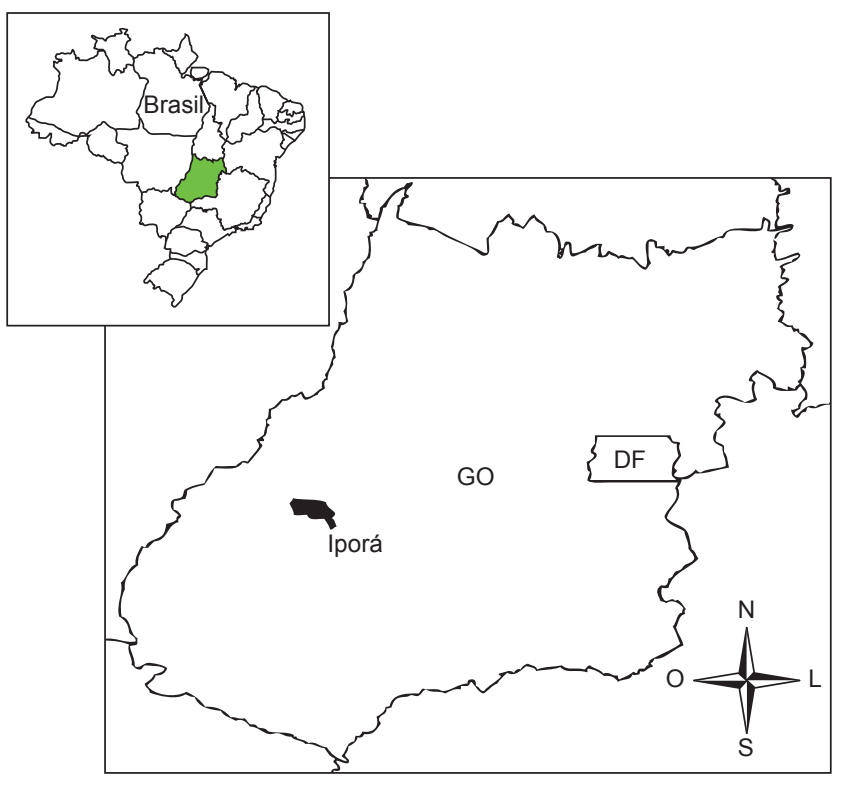

Figura 1. Localização do município de Iporá, Goiás, Brasil.

Figure 1. Location of Iporá, Goiás state, Brazil.

antrópica, uma vez que também é destinada ao lazer da população local (Cunha et al. 2007).

\section{Metodologia de coleta}

Dentro do Parque Municipal da Cachoeirinha, um espaço foi demarcado, compreendendo toda a região entre o córrego Tamanduá e o ribeirão Santo Antônio, com uma área total de aproximadamente $400 \mathrm{~m}$. Esse espaço foi escolhido por possuir uma vegetação, em sua maioria, rasteira e arbustiva, sendo mais propícia ao deslocamento e manejo da rede entomológica. Esta área era percorrida em toda sua extensão mesmo na ausência de flores. As coletas eram realizadas por duas horas diárias entre 6:30 e 18:30 horas, de acordo com as condições do tempo, não havendo coletas em dias chuvosos. O período de coleta foi de cinco dias entre o final de novembro e início de dezembro de 2004, 12 dias em março e 12 dias em maio de 2005, totalizando 58 horas de coleta. As abelhas foram coletadas com auxílio de rede entomológica através do método de varredura, capturando-se aquelas que estavam visitando flores ou em vôo sobre gramíneas e plantas em geral. As abelhas eram colocadas em sacolas plásticas e estas dentro de potes, onde eram anotados o horário e a subárea de coleta. Após o trabalho de campo, os espécimes foram acondicionados em mantas para posterior identificação, tendo sido utilizada a classificação Moure et al. (2008).

\section{Resultados e Discussão}

Foi coletado um total de 933 indivíduos distribuídos em uma família, 16 tribos, 33 gêneros e 62 espécies (Tabela 1). As espécies mais abundantes em ordem decrescente foram: Trigona chanchamayoensis (16,51\%), Tetragona clavipes (14,79\%), Trigona recursa $(12,33 \%)$ e Apis mellifera (12,33\%). As espécies mais abundantes observadas neste trabalho não representam uma surpresa, pois tanto as espécies de Meliponini mais abundantes (Tetragona clavipes, Trigona chanchamayoensis e Trigona recursa) quanto Apis mellifera podem apresentar colônias dispersas em uma mesma área devido aos seus hábitos de nidificação (Mateus 1998), o que pode ser uma explica- 
Espécies de abelhas em Iporá, GO

Tabela 1. Classificação taxonômica e número de abelhas coletadas no Parque Municipal da Cachoeirinha, Iporá, GO.

Table 1. Taxonomic classification and number of bees collected at Parque Municipal da Cachoeirinha (Iporá, Goiás state, Brazil).

\begin{tabular}{|c|c|c|c|c|c|c|}
\hline \multirow[t]{2}{*}{ Família } & \multirow[t]{2}{*}{ Subfamília } & \multirow[t]{2}{*}{ Tribo } & \multirow[t]{2}{*}{ Espécie } & \multicolumn{2}{|c|}{ Sexo } & \multirow[t]{2}{*}{ Total } \\
\hline & & & & M & $\mathbf{F}$ & \\
\hline Apidae & Andreninae & Oxaeini & Oxaea sp. & - & 1 & 1 \\
\hline Apidae & Apinae & Anthophorini & *Anthophora sp. & - & 1 & 1 \\
\hline Apidae & Apinae & Centridini & Centris (Centris) aenea (Lepeletier, 1841) & - & 1 & 1 \\
\hline Apidae & Apinae & Centridini & Centris (Trachina) fuscata (Lepeletier, 1841) & 4 & 4 & 8 \\
\hline Apidae & Apinae & Centridini & *Centris (Hemisiella) tarsata (Smith, 1874) & 1 & - & 1 \\
\hline Apidae & Apinae & Centridini & Centris (Hemisiella) trigonoides (Lepeletier, 1841) & 3 & 2 & 5 \\
\hline Apidae & Apinae & Centridini & ${ }^{*}$ Centris (Hemisiella) vittata (Lepeletier, 1841) & 1 & - & 1 \\
\hline Apidae & Apinae & Xylocopini & Ceratina sp.1 & - & 2 & 2 \\
\hline Apidae & Apinae & Xylocopini & Ceratina sp.2 & - & 1 & 1 \\
\hline Apidae & Apinae & Centridini & Epicharis (Epicharana) flava (Friese, 1900) & - & 1 & 1 \\
\hline Apidae & Apinae & Exomalopsini & Exomalopsis (Exomalopsis) auropilosa (Spinola, 1853) & - & 1 & 1 \\
\hline Apidae & Apinae & Exomalopsini & Exomalopsis (Exomalopsis) fulvofasciata (Smith, 1879) & - & 2 & 2 \\
\hline Apidae & Apinae & Eucerini & Melissoptila sp. & 2 & - & 2 \\
\hline Apidae & Apinae & Tapinotaspidini & Paratetrapedia connexa (Vachal, 1909) & 5 & 1 & 6 \\
\hline Apidae & Apinae & Tapinotaspidini & Paratetrapedia lugubris (Cresson, 1878) & 1 & - & 1 \\
\hline Apidae & Apinae & Tapinotaspidini & Paratetrapedia sp. nov.2 (Aguiar in litt) & - & 3 & 3 \\
\hline Apidae & Apinae & Tapinotaspidini & Paratetrapedia sp. nov.5 (Aguiar in litt) & - & 1 & 1 \\
\hline Apidae & Apinae & Tapinotaspidini & Paratetrapedia sp. nov.6 (Aguiar in litt) & - & 1 & 1 \\
\hline Apidae & Apinae & Tapinotaspidini & Paratetrapedia sp.1 & - & 1 & 1 \\
\hline Apidae & Apinae & Tapinotaspidini & Paratetrapedia sp.2 & - & 1 & 1 \\
\hline Apidae & Apinae & Tapinotaspidini & Paratetrapedia sp. 3 & 1 & - & 1 \\
\hline Apidae & Apinae & Tapinotaspidini & *Tapinotaspoides sp. & 2 & 2 & 4 \\
\hline Apidae & Apinae & Tetrapediini & Tetrapedia diversipes (Klug, 1810) & - & 1 & 1 \\
\hline Apidae & Apinae & Tetrapediini & Tetrapedia sp. & - & 2 & 2 \\
\hline Apidae & Apinae & Tetrapediini & Tetrapedia sp.1 & - & 2 & 2 \\
\hline Apidae & Apinae & Tetrapediini & Tetrapedia sp. 2 & 2 & - & 2 \\
\hline Apidae & Apinae & Xylocopini & Xylocopa (Neoxylocopa) cearensis (Ducke, 1910) & - & 1 & 1 \\
\hline Apidae & Apinae & Xylocopini & Xylocopa (Neoxylocopa) grisescens (Lepeletier, 1841) & - & 7 & 7 \\
\hline Apidae & Apinae & Xylocopini & Xylocopa (Schonnherria) muscaria (Fabricius, 1775) & - & 1 & 1 \\
\hline Apidae & Apinae & Apini & Apis mellifera (Linnaeus, 1758) & - & 115 & 115 \\
\hline Apidae & Apinae & Euglossini & Euglossa sp. & - & 2 & 2 \\
\hline Apidae & Apinae & Euglossini & Eulaema (Apeulaema) nigrita (Lepeletier, 1841) & - & 3 & 3 \\
\hline Apidae & Apinae & Meliponini & *Frieseomelitta silvestrii (Friese, 1902) & - & 1 & 1 \\
\hline Apidae & Apinae & Meliponini & Frieseomelitta varia (Lepeletier, 1836) & 1 & 6 & 7 \\
\hline Apidae & Apinae & Meliponini & *Oxytrigona $\mathrm{sp}$ & - & 1 & 1 \\
\hline Apidae & Apinae & Meliponini & Paratrigona lineata (Lepeletier, 1836) & - & 6 & 6 \\
\hline Apidae & Apinae & Meliponini & Partamona sp. & - & 20 & 20 \\
\hline Apidae & Apinae & Meliponini & *Plebeia sp. & - & 2 & 2 \\
\hline Apidae & Apinae & Meliponini & *Scaptotrigona depilis (Moure, 1942) & - & 7 & 7 \\
\hline Apidae & Apinae & Meliponini & Scaptotrigona cf. postica (Latreille, 1807) & - & 2 & 2 \\
\hline Apidae & Apinae & Meliponini & Scaptotrigona sp. & - & 1 & 1 \\
\hline Apidae & Apinae & Meliponini & *Scaura latitarsis (Friese, 1900) & - & 2 & 2 \\
\hline Apidae & Apinae & Meliponini & Scaura longula (Lepeletier, 1836) & - & 14 & 14 \\
\hline Apidae & Apinae & Meliponini & Tetragona clavipes (Fabricius, 1804) & - & 138 & 138 \\
\hline Apidae & Apinae & Meliponini & Tetragonisca angustula (Latreille, 1811) & - & 34 & 34 \\
\hline Apidae & Apinae & Meliponini & *Trigona chanchamayoensis (Schwarz, 1948) & - & 154 & 154 \\
\hline Apidae & Apinae & Meliponini & Trigona hyalinata (Lepeletier, 1836) & - & 56 & 56 \\
\hline Apidae & Apinae & Meliponini & Trigona fulviventris (Guérin, 1835) & 5 & 74 & 79 \\
\hline Apidae & Apinae & Meliponini & Trigona fuscipennis (Friese, 1900) & - & 85 & 85 \\
\hline
\end{tabular}


Tabela 1. Continuação...

\begin{tabular}{|c|c|c|c|c|c|c|}
\hline \multirow[t]{2}{*}{ Família } & \multirow[t]{2}{*}{ Subfamília } & \multirow[t]{2}{*}{ Tribo } & \multirow[t]{2}{*}{ Espécie } & \multicolumn{2}{|c|}{ Sexo } & \multirow[t]{2}{*}{ Total } \\
\hline & & & & M & $\mathbf{F}$ & \\
\hline Apidae & Apinae & Meliponini & Trigona recursa (Smith, 1863) & 1 & 114 & 115 \\
\hline Apidae & Apinae & Meliponini & Trigona spinipes (Fabricius, 1793) & - & 8 & 8 \\
\hline Apidae & Apinae & Meliponini & Trigona truculenta (Almeida, 1984) & - & 3 & 3 \\
\hline Apidae & Apinae & Meliponini & *Trigonisca nataliae (Moure, 1950) & - & 3 & 3 \\
\hline Apidae & Colletinae & Diphaglossini & Ptiloglossa sp. & - & 1 & 1 \\
\hline Apidae & Halictinae & Augochlorini & Augochlora sp.1 & - & 1 & 1 \\
\hline Apidae & Halictinae & Augochlorini & Augochlorella sp. & - & 1 & 1 \\
\hline Apidae & Halictinae & Augochlorini & Augochloropsis sp. 1 & 2 & 2 & 4 \\
\hline Apidae & Halictinae & Augochlorini & Augochloropsis sp.2 & - & 1 & 1 \\
\hline Apidae & Halictinae & Halictini & *Dialictus sp.1 & - & 1 & 1 \\
\hline Apidae & Halictinae & Augochlorini & Pseudaugochlora sp.1 & 1 & & 1 \\
\hline Apidae & Megachilinae & Anthidiini & Larocanthidium sp. & - & 1 & 1 \\
\hline \multirow[t]{2}{*}{ Apidae } & Megachilinae & Megachilini & Megachile sp. & - & 2 & 2 \\
\hline & & & & & & 933 \\
\hline
\end{tabular}

M: machos; F: fêmeas. *: registrado pela primeira vez em Goiás.

ção para a predominância destas quatro espécies. Contudo, o fato de Trigona chanchamayoensis ser a espécie predominante, Apis mellifera aparecer em terceiro lugar e Trigona spinipes ser muito pouco representada, foi um resultado inesperado, pois contrasta com outros trabalhos de abundância de espécies de abelhas uma vez que, por possuírem colônias muito populosas, Trigona spinipes e Apis mellifera costumam se apresentar como as espécies mais abundantes (Boaventura 1998, Mateus 1998, Aguiar \& Martins 2003, Vieira 2005, d'Avila \& Marchini 2008). Entretanto, a maior abundância de espécies do gênero Trigona já foi observada em áreas de Cerrado, por Rêgo \& Brito (1996), Mateus (1998), Boaventura \& Raw (1998), Santos et al. (2004) e Jesus (2007). A proximidade entre os ninhos também deve ser considerada, pois algumas abelhas apresentam comunicação intensa e específica ao encontrarem alimento, forrageando assim em grupo e influenciando o número de indivíduos coletados (Sakagami et al. 1967).

Até a presente publicação não havia registro da ocorrência dos gêneros Anthophora, Tapinotaspoides, Oxytrigona, Plebeia, Trigonisca e Dialictus no Estado de Goiás. Além disso, este também é o primeiro registro de ocorrência em Goiás para as espécies Centris (Hemisiella) tarsata, Centris (Hemisiella) vittata, Frieseomelitta silvestri, Scaptotrigona depilis, Scaura latitarsis e Trigona chanchamayoensis. Todos esses gêneros e espécies também podem ser encontrados em alguns estados que fazem fronteira com Goiás, mas chama a atenção o caso de Frieseomelitta silvestrii que até então só havia sido encontrada no Mato Grosso.

Apesar do Parque Municipal da Cachoeirinha (Iporá, GO) constituir-se em uma área de cerrado de pequena extensão e pobre em vegetação, o grande número de espécies de abelhas foi considerável se comparado a estudos de levantamento da apifauna realizados em áreas maiores e com grande número de horas de coleta. Tais resultados são surpreendentes, visto que a área estudada já foi muito alterada em relação à sua vegetação original, além de ainda estar sofrendo muito com a ação antrópica. A descrição da apifauna do Parque Municipal da Cachoeirinha realizada neste trabalho vem contribuir para o enriquecimento do conhecimento da diversidade de abelhas do Cerrado, em especial de Goiás, em virtude da ausência de estudos dessa natureza realizados no estado, e certamente abrirá caminho para que mais estudos de diversidade apícola sejam realizados na região.

\section{Agradecimentos}

Agradecemos aos revisores anônimos pelas valiosas sugestões para a melhoria deste artigo, à Elimárcia Maria da Silva pelo auxílio nas coletas, à Prefeitura Municipal de Iporá pela disponibilização da área de estudo e ao CNPq e à Universidade Estadual de Goiás pela bolsa de iniciação científica (PBIC/UEG) fornecida ao primeiro autor.

\section{Referências Bibliográficas}

AGUIAR, A.J.C. \& MARTINS, C.F. 2003. The bee diversity of the Tabuleiro vegetation in the Guaribas Biological Reserve (Mamanguape, Paraíba, Brazil). In Apoidea neotropica: homenagem aos 90 anos de Jesus Santiago Moure (G.A.R. Melo \& I. Alves dos Santos, eds). UNESC, Criciúma, p. 209-216.

ANACLETO, D.A. \& MARCHINI, L.C. 2005. Análise faunística de abelhas (Hymenoptera, Apoidea) coletadas no cerrado do estado de São Paulo. Acta Sci. Biol. Sci. 27(3):277-284.

ANDENA, S.R., BEGO, L.R. \& MECHI, R.M.A. 2005. Comunidade de abelhas (Hymenoptera, Apoidea) de uma área de cerrado (Corumbataí, SP) e suas visitas às flores. Rev. Bras. Zoocienc. 7(1):55-91.

BOAVENTURA, M.C. 1998. Sazonalidade e estrutura de uma comunidade de abelhas silvestres (Hymenoptera, Apoidea) numa área de cerrado do Jardim Botânico de Brasília, Distrito Federal. Dissertação de Mestrado, Universidade de Brasília, Brasília.

BOAVENTURA, M.C. \& RAW, A. 1998. Estrutura e recursos utilizados por uma comunidade de abelhas (Apoidea) em área de cerrado do Jardim Botânico de Brasília. In Anais do III Encontro sobre abelhas. USP, Ribeirão Preto, p. 260.

CARVALHO, A.M.C. \& BEGO, L.R. 1996. Studies on Apoidea fauna of cerrado vegetation at the Panga Ecological Reserve, Uberlândia, MG, Brazil. Rev. Bras. Entomol. 40(2):147-156.

CRISTOFOLI, M. \& LEÃO, L. 2005. Identificação parcial da flora do Parque Ecológico da Cachoeirinha - Iporá. Monografia, Universidade Estadual de Goiás, Iporá.

CUNHA, H.F., VALE, M.S., SILVA Jr., C.A., CAMPOS, R.F. \& CARLOS, L.O. 2007. Conhecimento empírico dos moradores da comunidade do entorno do Parque Municipal da Cachoeirinha (Iporá, Goiás). Acta Sci. Biol. 29(2):203-212. 
D'AVILA, M. \& MARCHINI, L.C. 2008. Análise faunística de himenópteros visitantes florais em fragmento de cerradão em Itirapina, SP. Cienc. Florest. 18(2):271-279.

JESUS, C.P., SILVA, F.M.A., ALBUQUERQUE, P.M.C. \& RÊGO, M.M.C. 2007. Abelhas silvestres em uma área de cerrado, Balsas - MA: composição faunística e fenologia. In Anais do VIII Congresso de Ecologia do Brasil. SEB, Caxambu, p. 1-2.

KERR, W.E., CARVALHO, G.A. \& NASCIMENTO, V.A. 1996. Abelha uruçu: biologia, manejo e conservação. Fundação Acangaú, Belo Horizonte.

LONGHI, E.H., OLIVEIRA, E., VANDERLEI, J.C., ROCHA, E.V. \& SILVA, I.D.C. 2005. Arranjo extrativista do pequi (Caryocar brasiliense Camb.) na região de Iporá, Goiás: sustentabilidade e dinâmica da comercialização. In XLIII Congresso da Sociedade Brasileira de Economia e Sociologia Rural. SOBER, Ribeirão Preto, p. 157.

MATEUS, S. 1998. Abundância relativa, fenologia e visita às flores pelos Apoidea do cerrado da estação ecológica de Jataí - Luiz Antônio, SP. Dissertação de Mestrado, Universidade de São Paulo, Ribeirão Preto.

MICHENER, C.D. 2000. The bees of the World. The Johns Hopkins University Press, Baltimore and London.

MOURE, J.S., URBAN, D. \& MELO, G.A.R. 2008. Catalogue of bees (Hymenoptera, Apoidea) in the Neotropical Region. http://www.moure. cria.orgbr/catalogue (último acesso em 05/07/2009).

NOGUEIRA-NETO, P. 1997. Vida e criação de abelhas indígenas sem ferrão. Nogueirapis, São Paulo.

PEDRO, S.R.M. 1992. Sobre as abelhas (Hymenoptera, Apoidea) em um ecossistema de cerrado (Cajuru, NE do Estado de São Paulo): composição, fenologia e visita às flores. Dissertação de Mestrado Universidade de São Paulo, Ribeirão Preto.

RATTER, J.A., RIBEIRO, J.F. \& BRIDGEWATER, S. 1997. The brazilian cerrado vegetation and threats to its biodiversity. Ann. Bot. 80(3):223230 .
RÊGO, M. \& BRITO, C. 1996. Meliponíneos em um ecossistema de cerrado s.l. (Leste do Maranhão, BR). In Anais do III Encontro sobre abelhas. USP, Ribeirão Preto, p. 278.

SAKAGAMI, S.F., LAROCA, S. \& MOURE, J.S. 1967. Wild bee biocoenotics in São José dos Pinhais (PR), South Brazil: preliminary report. J. Fac. Sci. 16(2):252-291.

SANTOS, M.F., CARVALHO, C.A.L. \& SILVA, R.F. 2004. Diversidade de abelhas (Hymenoptera: Apoidea) em uma área de transição CerradoAmazônia. Acta Amazon. 34(2):319-328.

SILVA, M.A.C., FLEURI-JARDIM, A.V. \& IGUATEMY, M.A. 2005. Riqueza e abundância de espécies em quatro fragmentos de cerrado do interior paulista ao longo de quatro anos de estudo, SP. In Relatórios de projetos desenvolvidos na disciplina NE211, Ecologia de Campo II do Programa de Pós-Graduação em Ecologia da Universidade Estadual de Campinas. (F.A.M. Santos, F.R. Martins, J.Y. Tamashiro, orgs). http:// www.ib.unicamp.br/profs/fsantos/ecocampo/ne211/2005/ (último acesso em 10/12/2008).

SILVEIRA, F.A. \& CAMPOS, M.J.O. 1995. A melissofauna de Corumbataí (SP) e Paraopeba (MG) e uma análise da biogeografia das abelhas do cerrado brasileiro (Hymenoptera, Apoidea). Rev. Bras. Entomol. 39(2):371-401.

SILVEIRA, F.A. \& GODÍNEZ, L.M. 1996. Systematics surveys of local bee faunas. Melissa. 9:1-4.

VIEIRA, G.H.C. 2005. Análise faunística de abelhas (Hymenoptera: Apoidea) e tipificação dos méis produzidos por Apis mellifera L., em área de cerrado no município de Cassilândia/MS. Tese de Doutorado, Universidade de São Paulo, Piracicaba.

VIEIRA, G.H.C., MARCHINI, L.C., SOUZA, B.A. \& MORETI, C.C.A. 2008. Fontes florais usadas por abelhas (Hymenoptera, Apoidea) em área de cerrado no Município de Cassilândia, Mato Grosso do Sul, Brasil. Cienc. Agrotec. 32(5):1454-1460.

Recebido em 02/02/09

Versão reformulada recebida em 07/07/09

Publicado em 10/07/09 